\title{
On topological groups with an approximate fixed point property
}

\author{
CLEON S. BARROSO ${ }^{1}$, BRICE R. MBOMBO ${ }^{2,4}$ and VLADIMIR G. PESTOV ${ }^{3,4}$ \\ ${ }^{1}$ Departamento de Matemática, Universidade Federal do Ceará, Campus do Pici, B1. 914, 60455-760 Fortaleza, CE, Brazil \\ ${ }^{2}$ Departamento de Matemática, Instituto de Matemática e Estatística, Universidade de São Paulo, Rua do Matão, 1010, 05508-090 \\ São Paulo, SP, Brazil \\ ${ }^{3}$ Departamento de Matemática, Universidade Federal de Santa Catarina, Trindade, 88040-900 Florianópolis, SC, Brazil \\ ${ }^{4}$ Department of Mathematics and Statistics, University of Ottawa, Ottawa, ON, K1N 6N5, Canada
}

Manuscript received on June 25, 2015; accepted for publication on October 10, 2016

\begin{abstract}
A topological group $G$ has the Approximate Fixed Point (AFP) property on a bounded convex subset $C$ of a locally convex space if every continuous affine action of $G$ on $C$ admits a net $\left(x_{i}\right), x_{i} \in C$, such that $x_{i}-g x_{i} \longrightarrow 0$ for all $g \in G$. In this work, we study the relationship between this property and amenability.
\end{abstract}

Key words: Amenable groups, approximate fixed point property, Følner property, Reiter property.

\section{INTRODUCTION}

One of the most useful known characterizations of amenability is stated in terms of a fixed point property. A classical theorem of (Day 1961) says that a topological group $G$ is amenable if and only if every continuous affine action of $G$ on a compact convex subset $C$ of a locally convex space has a fixed point, that is, a point $x \in C$ with $g \cdot x=x$ for all $g \in G$. This result generalizes earlier theorems of (Kakutani 1938) and (Markov 1936) obtained for abelian acting groups.

At the same time, an active branch of current research is devoted to the existence of approximate fixed points for single maps. Basically, given a bounded, closed convex set $C$ and a map $f: C \longrightarrow C$, one wants to find a sequence $\left(x_{n}\right) \subset C$ such that $x_{n}-f\left(x_{n}\right) \longrightarrow 0$. A sequence with this property will be called an approximate fixed point sequence.

The main motivation for this topic is purely mathematical and comes from several instances of the failure of the fixed point property in convex sets that are no longer assumed to be compact, cf. (Dobrowolski and Marciszewski 1997, Edelstein and Kok-Keong 1994, Floret 1980, Klee 1955, Nowak 1979) and references therein. One of the most emblematic results on this matter states that if $C$ is a non-compact,

AMS Classification: 47-03, 47H10, 54H25, 37B05.

Correspondence to: Brice R. Mbombo

E-mail: brice@ime.usp.br 
bounded, closed convex subset of a normed space, then there exists a Lipschitz map $f: C \longrightarrow C$ such that $\inf _{x \in C}\|x-f(x)\|>0$ (Lin and Sternfeld 1985). Notice in this case that there is no approximate fixed point sequence for $f$. Previous results of topological flavour were discovered by many authors, including (Klee 1955) who has characterized the fixed point property in terms of compactness in the framework of metrizable locally convex spaces.

Both results give rise to the natural question whether a given space without the fixed point property might still have the approximate fixed point property. The first thoughts on this subject were developed in (Barroso 2009, Barroso and Lin 2010, Barroso et al. 2012, 2013) in the context of weak topologies. Another mathematical motivation for the study of the approximate fixed point property is the following open question.

Question 0.1. Let $X$ be a Hausdorff locally convex space. Assume $C \subset X$ is a sequentially compact convex set and $f: C \longrightarrow C$ is a sequentially continuous map. Does $f$ have a fixed point?

So far, the best answers for this question were delivered in (Barroso et al. 2012, 2013). Let us just summarize the results.

Theorem 0.2 (Theorem 2.1, Proposition 2.5(i) in (Barroso et al. 2012, 2013)). Let $X$ be a topological vector space, $C \subset X$ a nonempty convex set, and let $f: C \longrightarrow \bar{C}$ be a map.

1. If $C$ is bounded, $f(C) \subset C$ and $f$ is affine, then $f$ has an approximate fixed point sequence.

2. If $X$ is locally convex and $f$ is sequentially continuous with totally bounded range, then $0 \in$ $\overline{\{x-f(x): x \in C\}}$. And indeed, $f$ has a fixed point provided that $X$ is metrizable.

3. If $C$ is bounded and $f$ is $\tau$-to- $\sigma(X, Z)$ sequentially continuous, then $0 \in \overline{\{x-f(x): x \in C\}}^{\sigma(X, Z)}$, where $\tau$ is the original topology of $X$ and $Z$ is a subspace of the topological dual $X^{\star}$. And, moreover, $f$ has a $\sigma(X, Z)$-approximate fixed point sequence provided that $Z$ is separable under the strong topology induced by $X$.

The idea of approximate fixed points is an old one. Apparently the first result on this kind was exploited in (Scarf 1967), where a constructive method for computing fixed points of continuous mappings of a simplex into itself was described. Other important works along these lines can be found in Hazewinkel and van de Vel (1978), Hadžić (1996), Idzik (1988), Park (1972). Approximate fixed point property has a lot of applications in many interesting problems. In (Kalenda 2011) it is proved that a Banach space has the weak-approximate fixed point property if and only if it does not contain any isomorphic copy of $\ell^{1}$. As another instance, it can be used to study the existence of limiting-weak solutions for differential equations in reflexive Banach spaces (Barroso 2009).

In this note, we study the existence of common approximate fixed points for a set of transformations forming a topological group. Not surprisingly, the approximate fixed point property for an acting group $G$ is also closely related to amenability of $G$, however, the relationship appears to be more complex.

There is an extremely broad variety of known definitions of amenability of a topological group, which are typically equivalent in the context of locally compact groups yet may diverge beyond this class. One would expect the approximate fixed point property (or rather "properties," for they depend on the class of convex sets allowed) to provide a new definition of amenability for some class of groups, and delineate a new class of topological groups in more general contexts. This indeed turns out to be the case. 
We show that a discrete group $G$ is amenable if and only if every continuous affine action of $G$ on a bounded convex subset $C$ of a locally convex space (LCS) $X$ admits approximate fixed points. For a locally compact group, a similar result holds if we consider actions on bounded convex sets $C$ which are complete in the additive uniformity, while in general we can only prove that $G$ admits weakly approximate fixed points. This criterion of amenability is no longer true in the more general case of a Polish group, even if amenability of Polish groups can be expressed in terms of the approximate fixed point property on bounded convex subsets of the Hilbert space.

We view our investigation as only the first step in this direction, and so we close the article with a discussion of open problems for further research.

\section{1 - AMENABILITY}

Here is a brief reminder of some facts about amenable topological groups. For a more detailed treatment, see e.g. (Paterson 1988). All the topologies considered here are assumed to be Hausdorff.

Let $G$ be a topological group. The right uniform structure on $G$ has as basic entourages of the diagonal the sets of the form $U_{V}=\left\{(g, h) \in G \times G \mid h g^{-1} \in V\right\}$, where $V$ is a neighbourhood of the identity $e$ in $G$. This structure is invariant under right translations. Accordingly, a function $f: G \longrightarrow \mathbb{R}$ is right uniformly continuous if for all $\varepsilon>0$, there exists a neighbourhood $V$ of $e$ in $G$ such that $x y^{-1} \in V$ implies $|f(x)-f(y)|<\varepsilon$ for every $x, y \in G$. Let $R U C B(G)$ denote the space of all right uniformly continuous functions equipped with the uniform norm. The group $G$ acts on $\operatorname{RUCB}(G)$ on the left continuously by isometries: for all $g \in G$ and $f \in R U C B(G), g \cdot f={ }^{g} f$ where ${ }^{g} f(x)=f\left(g^{-1} x\right)$ for all $x \in G$.

Definition 1.1. A topological group $G$ is amenable if it admits an invariant mean on $\operatorname{RUCB}(G)$, that is, a positive linear functional $\mathrm{m}$ with $\mathrm{m}(1)=1$, invariant under the left translations.

Examples of such groups include finite groups, solvable topological groups (including nilpotent, in particular abelian topological groups) and compact groups. Here are some more examples:

1. The unitary group $\mathcal{U}\left(\ell^{2}\right)$, equipped with the strong operator topology (de la Harpe 1973).

2. The infinite symmetric group $S_{\infty}$ with its unique Polish topology.

3. The group $\mathcal{J}(\mathrm{k})$ of all formal power series in a variable $x$ that have the form $f(x)=x+\alpha_{1} x^{2}+$ $\alpha_{2} x^{3}+\ldots, \quad \alpha_{n} \in \mathrm{k}$, where $\mathrm{k}$ is a commutative unital ring (Babenko and Bogatyi 2011).

Let us also mention some examples of non-amenable groups:

1. The free discrete group $\mathbb{F}_{2}$ of two generators. More generally, every locally compact group containing $\mathbb{F}_{2}$ as a closed subgroup.

2. The unitary group $\mathcal{U}\left(\ell^{2}\right)$, with the uniform operator topology (de la Harpe 1979).

3. The group $\operatorname{Aut}(X, \mu)$ of all measure-preserving automorphisms of a standard Borel measure space $(X, \mu)$, with the uniform topology, i.e. the topology determined by the metric $d(\tau, \sigma)=\mu\{x \in X$ : $\tau(x) \neq \sigma(x)\}$ (Giordano and Pestov 2002).

The following is one of the main criteria of amenability in the locally compact case. 
Theorem 1.2 (Følner's condition). Let $G$ be a locally compact group and denote $\lambda$ the left invariant Haar measure. Then $G$ is amenable if and only if $G$ satisfies the Følner condition: for every compact set $F \subseteq G$ and $\varepsilon>0$, there is a Borel set $U \subseteq G$ of positive finite Haar measure $\lambda(U)$ such that $\frac{\lambda(x U \triangle U)}{\lambda(U)}<\varepsilon$ for each $x \in F$.

Recall that a Polish group is a topological group whose topology is Polish, i.e., separable and completely metrizable.

Proposition 1.3 (See e.g. (Al-Gadid et al. 2011), Proposition 3.7). A Polish group $G$ is amenable if and only if every continuous affine action of $G$ on a convex, compact and metrizable subset $K$ of a locally convex space $X$ admits a fixed point.

For a most interesting recent survey about the history of amenable groups, see (Grigorchuk and de la Harpe in press).

\section{2 - GROUPS WITH APPROXIMATE FIXED POINT PROPERTY}

Definition 2.1. Let $C$ be a convex bounded subset of a topological vector space $X$. Say that a topological group $G$ has the approximate fixed point (AFP) property on $C$ if every continuous affine action of $G$ on $C$ admits an approximate fixed point net, that is, a net $\left(x_{i}\right) \subseteq C$ such that for every $g \in G, x_{i}-g x_{i} \longrightarrow 0$.

We will analyse the AFP property of various classes of amenable topological groups.

\section{1 - CASE OF DISCRETE GROUPS}

Theorem 2.2. The following properties are equivalent for a discrete group $G$ :

1. G is amenable,

2. G has the AFP property on every convex bounded subset of a locally convex space.

Proof. (1) $\Rightarrow(2)$. Let $G$ a discrete amenable group acting by continuous affine maps on a bounded convex subset $C$ of a locally convex space $X$. Choose a Følner's $\left(\Phi_{i}\right)_{i \in I}$ net, that is, a net of finite subsets of $G$ such that

$$
\frac{\left|g \Phi_{i} \triangle \Phi_{i}\right|}{\left|\Phi_{i}\right|} \longrightarrow 0 \quad \forall g \in G .
$$


Now, let $\gamma \in G$, fix $x \in C$ and define $x_{i}=\frac{1}{\left|\Phi_{i}\right|} \sum_{g \in \Phi_{i}} g x$. Since $C$ is convex, $x_{i} \in C$ for all $i \in I$. Notice that $\left|\Phi_{i} \backslash \gamma \Phi_{i}\right|=\left|\gamma \Phi_{i} \backslash \Phi_{i}\right|=\frac{1}{2}\left|\gamma \Phi_{i} \triangle \Phi_{i}\right|$ for all $i \in I$. Therefore we have

$$
\begin{aligned}
x_{i}-\gamma x_{i} & =\frac{1}{\left|\Phi_{i}\right|}\left[\sum_{g \in \Phi_{i}} g x-\sum_{g \in \Phi_{i}} \gamma g x\right] \\
& =\frac{1}{\left|\Phi_{i}\right|}\left[\sum_{g \in \Phi_{i}} g x-\sum_{h \in \gamma \Phi_{i}} h x\right] \\
& =\frac{1}{\left|\Phi_{i}\right|}\left[\sum_{g \in\left(\Phi_{i} \backslash \gamma \Phi_{i}\right)} g x-\sum_{g \in\left(\gamma \Phi_{i} \backslash \Phi_{i}\right)} g x\right] \\
& =\frac{\left|\gamma \Phi_{i} \triangle \Phi_{i}\right|}{2\left|\Phi_{i}\right|}\left[\frac{1}{\left|\Phi_{i} \backslash \gamma \Phi_{i}\right|} \sum_{g \in\left(\Phi_{i} \backslash \gamma \Phi_{i}\right)} g x-\frac{1}{\left|\gamma \Phi_{i} \backslash \Phi_{i}\right|} \sum_{g \in\left(\gamma \Phi_{i} \backslash \Phi_{i}\right)} g x\right]
\end{aligned}
$$

Thus $x_{i}-\gamma x_{i} \in \frac{\left|\gamma \Phi_{i} \triangle \Phi_{i}\right|}{2\left|\Phi_{i}\right|}(C-C)$ and hence $x_{i}-\gamma x_{i} \longrightarrow 0$ since $C$ is bounded.

$(2) \Rightarrow(1)$. Let $G$ be a discrete group acting continuously and by affine transformations on a nonempty compact and convex set $K$ in a locally convex space $X$. By hypothesis, there is a net $\left(x_{i}\right) \subseteq K$ such that $\forall g \in G, x_{i}-g x_{i} \longrightarrow 0$. By compactness of $K$, this net has accumulation points in $K$. Since $\forall g \in$ $G, x_{i}-g x_{i} \longrightarrow 0$, this insures invariance of accumulation points and shows the existence of a fixed point in $K$. Therefore $G$ is an amenable group by Day's fixed point theorem mentioned in the Introduction.

\section{2 - CASE OF LOCALLY COMPACT GROUPS}

Recall from (Bourbaki 1963) the following notion of integration of functions with range in a locally convex space.

Let $F$ be a locally convex vector space on $\mathbb{R}$ or $\mathbb{C} . F^{\prime}$ denotes the dual space of $F$ and $F^{\prime *}$ the algebraic dual of $F^{\prime}$. We identify as usual $F$ (seen as a vector space without topology) with a subspace of $F^{\prime *}$ by associating to any $z \in F$ the linear form $F^{\prime} \ni z^{\prime} \longmapsto\left\langle z, z^{\prime}\right\rangle \in \mathbb{R}$.

Let $T$ be a locally compact space and let $\mu$ a positive measure on $T$. A map $f: T \longrightarrow F$ is essentially $\mu$-integrable if for every element $z^{\prime} \in F^{\prime},\left\langle z^{\prime}, f\right\rangle$ is essentially $\mu$-integrable. If $f: T \longrightarrow F$ is essentially $\mu$-integrable, then $z^{\prime} \longmapsto \int_{T}\left\langle z^{\prime}, f\right\rangle \mathrm{d} \mu$ is a linear map on $F^{\prime}$, i.e. an element of $F^{* *}$. The integral of $f$ is the element of $F^{*}$ denoted $\int_{T} f \mathrm{~d} \mu$ and defined by the condition: $\left\langle z^{\prime}, \int_{T} f \mathrm{~d} \mu\right\rangle=\int_{T}\left\langle z^{\prime}, f\right\rangle \mathrm{d} \mu$ for every $z^{\prime} \in F^{\prime}$.

Note that, in general we don't have $\int_{T} f \mathrm{~d} \mu \in F$. But we have the following. 
Proposition 2.3 ((Bourbaki 1963), chap. 3, Proposition 7). Let $T$ be a locally compact space, E a locally convex space and $f: T \longrightarrow E$ a function with compact support. If $f(T)$ is contained in a complete (with regard to the additive uniformity) convex subset of $E$, then $\int_{T} f d \mu \in E$.

Theorem 2.4. The following are equivalent for a locally compact group $G$ :

1. G is amenable,

2. G has the AFP property on every complete, convex, and bounded subset of a locally convex space.

Proof. (1) $\Rightarrow(2)$. Let $G$ be a locally compact amenable group acting continuously by affine maps on a complete, bounded, convex subset $C$ of a locally convex space $X$. Again, select a Følner net $\left(F_{i}\right)_{i \in I}$ of compact subsets of $G$ such that $\frac{\lambda\left(g F_{i} \triangle F_{i}\right)}{\lambda\left(F_{i}\right)} \longrightarrow 0 \quad \forall g \in G$. Fix $x \in C$ and let $\eta_{x}: G \ni g \longmapsto g x \in C$ be the corresponding orbit map. Define $x_{i}=\frac{1}{\lambda\left(F_{i}\right)} \int_{F_{i}} \eta_{x}(g) \mathrm{d} \lambda(g)$. By the above, this is an element of $C$; the barycenter of the push-forward measure $\left(\eta_{x}\right)_{*}\left(\left.\lambda\right|_{F_{i}}\right)$ on $X$. We have, just like in the discrete case:

$$
\begin{aligned}
x_{i}-\gamma x_{i} & =\frac{1}{\lambda\left(F_{i}\right)}\left[\int_{F_{i}} \eta_{x}(g) \mathrm{d} \lambda(g)-\gamma \int_{F_{i}} \eta_{x}(g) \mathrm{d} \lambda(g)\right] \\
& =\frac{1}{\lambda\left(F_{i}\right)}\left[\int_{F_{i}} \eta_{x}(g) \mathrm{d} \lambda(g)-\int_{F_{i}} \eta_{x}(\gamma g) \mathrm{d} \lambda(g)\right] \\
& =\frac{1}{\lambda\left(F_{i}\right)}\left[\int_{F_{i}}\left[\eta_{x}(g)-\eta_{x}(\gamma g)\right] \mathrm{d} \lambda(g)\right] \\
& =\frac{1}{\lambda\left(F_{i}\right)}\left[\int_{\gamma F_{i} \triangle F_{i}} \eta_{x}(v) \mathrm{d} \lambda(v)\right] .
\end{aligned}
$$

Now, let $q$ be any continuous seminorm on $C$. We have:

$$
q\left(x_{i}-\gamma x_{i}\right) \leq \frac{\lambda\left(\gamma F_{i} \triangle F_{i}\right)}{\lambda\left(F_{i}\right)} K
$$

where $K=\sup _{v \in G} q \circ \eta_{x}(v)<\infty$ since $C$ is bounded. Thus $x_{i}-\gamma x_{i} \longrightarrow 0$.

$(2) \Rightarrow(1)$. Same argument as in the case of discrete groups.

The assumption of completeness of $C$ does not look natural in the context of approximate fixed points, but we do not know if it can be removed. It depends on the answer to the following.

Question 2.5. Let $f$ be an affine homeomorphism of a bounded convex subset $C$ of a locally convex space $X$. Can $f$ be extended to a continuous map (hence, a homeomorphism) of the closure of $C$ in $X$ ? 
Nevertheless, we can prove the following.

Theorem 2.6. Every amenable locally compact group $G$ has a weak approximate fixed point property on each bounded convex subset $C$ of a locally convex space $X$.

Proof. In the notation of the proof of Theorem 2.4, let $\mu_{i}=\left(\eta_{x}\right)_{*}\left(\left.\lambda\right|_{F_{i}}\right)$ denote the push-forward of the measure $\lambda_{i}=\lambda \uparrow F_{i}$ along the orbit map $\eta_{x}: G \ni g \longmapsto g x \in C$. Let $x_{i}$ be the barycenter of $\mu_{i}$. This time, $x_{i}$ need not belong to $C$ itself, but will belong to the completion $\hat{C}$, of $C$ (the closure of $C$ in the locally convex vector space completion $\hat{X}$ ).

For every $g \in G$, denote $z_{i}^{g}$ the barycenter of the measure $g \cdot \mu_{i}=\left(\eta_{x}\right)_{*}\left(g \lambda_{i}\right)$. Just like in the proof of Theorem 2.4, for every $g$ we have $x_{i}-z_{i}^{g} \rightarrow 0$.

Now select a net $\nu_{j}$ of measures with finite support on $G$, converging to $\lambda_{i}$ in the vague topology (Bourbaki 1963). Denote $y_{j}$ the barycenter of the push-forward measure $\left(\eta_{x}\right)_{*}\left(\nu_{j}\right)$. Then $y_{j} \Rightarrow x_{i}$ in the vague topology on the space of finite measures on the compact space $F_{i} . x$. Clearly, $y_{j} \in C$, and so $g \cdot y_{j}$ is well-defined and $g \cdot y_{j} \Rightarrow z_{i}^{g}$ for every $g \in \Phi$. It follows that $g \cdot y_{j}-y_{j}$ weakly converges to 0 for every $g \in \Phi$.

Remark 2.7. Clearly the weak AFP property on each bounded convex subset $C$ implies amenability of $G$ as well. Recall that a topological group $G$ has the weak AFP property on $C$ if every weakly continuous affine action of $G$ on $C$ admits an approximate fixed point net.

\section{3 - CASE OF POLISH GROUPS}

The above criteria do not generalize beyond the locally compact case in the ways one might expect: not every amenable non-locally compact Polish group has the AFP property, even on a bounded convex subset of a Banach space.

Proposition 2.8. The infinite symmetric group $S_{\infty}$ equipped with its natural Polish topology does not have the AFP property on closed convex bounded subsets of $\ell^{1}$.

If we think of $S_{\infty}$ as the group of all self-bijections of the natural numbers $\mathbb{N}$, then the natural (and only) Polish topology on $S_{\infty}$ is induced from the embedding of $S_{\infty}$ into the Tychonoff power $\mathbb{N}^{\mathbb{N}}$, where $\mathbb{N}$ carried the discrete topology.

We will use the following well-known criterion of amenability for locally compact groups.

Theorem 2.9 (Reiter's condition). Let $p$ be any real number with $1 \leq p<\infty$. A locally compact group $G$ is amenable if and only if for any compact set $C \subseteq G$ and $\varepsilon>0$, there exists $f \in L^{p}(G), f \geq 0,\|f\|_{p}=1$, such that: $\|g \cdot f-f\|<\varepsilon$ for all $g \in C$.

Proof of Proposition 2.8. Denote $\operatorname{prob}(\mathbb{N})$ the set of all Borel probability measures on $\mathbb{N}$, in other words, the set of positive functions $b: \mathbb{N} \longrightarrow[0,1]$ such that $\sum_{n \in \mathbb{N}} b(n)=1$. This is the intersection of the unit sphere of $\ell^{1}$ with the cone of positive elements, a closed convex bounded subset of $\ell^{1}$. The Polish group $S_{\infty}$ acts canonically on $\ell^{1}$ by permuting the coordinates:

$$
S_{\infty} \times \ell^{1}(\mathbb{N}) \ni\left(\sigma,\left(x_{n}\right)_{n}\right) \longmapsto \sigma \cdot\left(x_{n}\right)_{n}=\left(x_{\sigma^{-1}(n)}\right)_{n} \in \ell^{1}(\mathbb{N}) .
$$


Clearly, $\operatorname{prob}(\mathbb{N})$ is invariant and the restricted action is affine and continuous. We will show that the action of $S_{\infty}$ on $\operatorname{prob}(\mathbb{N})$ admits no approximate fixed point sequence.

Assume the contrary. Make the free group $\mathbb{F}_{2}$ act on itself by left multiplication and identify $\mathbb{F}_{2}$ with $\mathbb{N}$. In this way we embed $\mathbb{F}_{2}$ into $S_{\infty}$ as a closed discrete subgroup. This means that the action of $\mathbb{F}_{2}$ by left regular representation on $\operatorname{prob}(\mathbb{N}) \cong \operatorname{prob}\left(\mathbb{F}_{2}\right)$ also has almost fixed points, and $\ell^{1}\left(\mathbb{F}_{2}\right)$, with regard to the left regular representation of $\mathbb{F}_{2}$, has almost invariant vectors. But this is the Reiter's condition $(p=1)$ for $\mathbb{F}_{2}$, a contradiction with non-amenability of this group.

However, it is still possible to characterize amenability of Polish groups in terms of the AFP property.

Theorem 2.10. The following are equivalent for a Polish group $G$ :

1. G is amenable,

2. G has the AFP property on every bounded, closed and convex subset of the Hilbert space.

Proof. (1) $\Rightarrow$ (2). It is enough to show that a norm-continuous affine action of $G$ on a bounded closed convex subset $C$ of $\ell^{2}$ is continuous with regard to the weak topology, because then there will be a fixed point in $C$ by Day's theorem.

Let $x \in C$ and $g \in G$ be any, and let $V$ be a weak neighbourhood of $g . x$ in $C$. The weak topology on the weakly compact set $C$ coincides with the $\sigma\left(\operatorname{span} C, \ell^{2}\right)$ topology, hence one can choose $x_{1}, x_{2}, \ldots, x_{n} \in C$ and $\varepsilon>0$ so that $y \in V$ whenever $\left|\left\langle x_{i}, y-g x\right\rangle\right|<\varepsilon$ for all $i$.

Denote $K$ the diameter of $C$. Because the action is norm-continuous, we can find $U \ni e$ in $G$ so that $\left\|u^{-1} x_{i}-x_{i}\right\|<\varepsilon / 2 K$ for all $i$. The set $U g$ is a neighbourhood of $g$ in $G$.

As a weak neighbourhood of $x$, take the set $W$ formed by all $\zeta \in C$ with $\left|\left\langle g^{-1} x_{i}, \zeta-x\right\rangle\right|<\varepsilon / 2$ for all $i$. Equivalently, the condition on $\zeta$ can be stated $\left|\left\langle x_{i}, g \zeta-g x\right\rangle\right|<\varepsilon / 2$ for all $i$.

If now $u \in U$ and $\zeta \in W$, one has

$$
\begin{aligned}
\left|\left\langle x_{i},(u g) \cdot \zeta-g x\right\rangle\right| & =\left|\left\langle u^{-1} x_{i}, g \zeta\right\rangle-\left\langle x_{i}, g x\right\rangle\right| \\
& =\left|\left\langle u^{-1} x_{i}, g \zeta\right\rangle-\left\langle x_{i}, g \zeta\right\rangle\right|+\left|\left\langle x_{i}, g \zeta\right\rangle-\left\langle x_{i}, g x\right\rangle\right| \\
& \leq\left\|u x_{i}-x_{i}\right\| \cdot K+\frac{\varepsilon}{2} \\
& =\varepsilon .
\end{aligned}
$$

This shows that $(u g) \cdot \zeta \in V$, and so the action of $G$ on $C$ is continuous with regard to the weak topology.

$(2) \Rightarrow(1)$. Suppose that $G$ acts continuously and by affine transformations on a compact convex and metrizable subset $Q$ of a LCS $E$. If $C(Q)$ is equipped with the usual norm topology, then $G$ acts continuously by affine transformations on the subspace $A(Q)$ of $C(Q)$ consisting of affine continuous functions on $Q$. Since $Q$ is a metrizable compact set, the space $C(Q)$ is separable, so is the space $A(Q)$. Fix a dense countable subgroup $H$ of $G$, and let $F=\left\{f_{n}: n \in \mathbb{N}\right\}$ be a dense subset of the closed unit ball of $A(Q)$ which is $H$-invariant. The map $T: Q \ni x \longmapsto\left(\frac{1}{n} f_{n}(x)\right) \in \ell^{2}$ is an affine homeomorphism of $Q$ onto a convex compact subset of $\ell^{2}$. The subgroup $H$ acts continuously and by affine transformations on the affine topological copy $T(Q)$ of $Q$ by, the obvious rule $h \cdot T(x)=T(h \cdot x)$. The action of $H$ extends by continuity 
to a continuous affine action of $G$ on $T(Q)$. By hypothesis, $G$ admits an approximative fixed point sequence in $T(Q)$, and every accumulation point of this sequence is a fixed point since $Q$ is compact. Therefore $G$ is amenable.

\section{3 - DISCUSSION AND CONCLUSION}

\section{1 - APPROXIMATELY FIXED SEQUENCES}

As we have already noted, we do not know if every locally compact amenable group has the AFP property on all convex bounded subsets of locally convex spaces. Another interesting problem is to determine when does an acting group possess not merely an approximately fixed net, but an approximate fixed sequence.

Recall that a topological group $G$ is $\sigma$-compact if it is a union of countably many compact subsets. It is easy to see that if an amenable locally compact group $G$ is $\sigma$-compact, then it admits an approximate fixed sequence for every continuous action by affine maps on a closed bounded convex set.

Question 3.1. Let $G$ be a metrizable separable group acting continuously and affinely on a convex bounded subset $C$ of a metrizable and locally convex space. If the action has an approximate fixed net, does there necessarily exist an approximate fixed sequence?

This is the case, for example if $G$ is the union of a countable chain of amenable locally compact (in particular, compact) subgroups, and the convex set $C$ is complete.

Recall in this connection that amenability (and thus Day's fixed point property) is preserved by passing to the completion of a topological group. At the same time, the AFP property is not preserved by completions. Indeed, the group $S_{\infty}^{f i n}$ of all permutations of integers with finite support is amenable as a discrete group, and so, equipped with any group topology, will have the AFP property on every bounded convex subset of a locally convex space. However, its completion with regard to the pointwise topology is the Polish group $S_{\infty}$ which, as we have seen, fails the AFP property on a bounded convex subset of $\ell^{1}$.

Question 3.2. Does every amenable group whose left and right uniformities are equivalent (a SIN group) have the AFP property on complete convex sets?

\section{2 - DISTAL ACTIONS}

Let $G$ be a topological group acting by homeomorphisms on a compact set $Q$. The flow $(G, Q)$ is called distal if whenever $\lim _{\alpha} s_{\alpha} \cdot x=\lim _{\alpha} s_{\alpha} \cdot y$ for some net $s_{\alpha}$ in $G$, then $x=y$. A particular class of distal flows is given by equicontinuous flows, for which the collection of all maps $x \mapsto g \cdot x$ forms an equicontinuous family on the compact space $Q$. We have the following fixed point theorem:

Theorem 3.3 (Hahn 1967). If a compact affine flow $(G, Q)$ is distal, then there is a G-fixed point.

An earlier result by (Kakutani 1938) established the same for the class of equicontinuous flows.

Question 3.4. Is there any approximate fixed point analogue of the above results for distal or equicontinuous actions by a topological group on a (non-compact) bounded convex set $Q$ ? 


\section{3 - NON-AFFINE MAPS}

Historically, Day's theorem (and before that, the theorem of Markov and Kakutani) was inspired by the classical Brouwer fixed point theorem (Brouwer 1911) and its later more general versions, first for Banach spaces (Schauder 1930) and later for locally convex linear Hausdorff topological spaces (Tychonoff 1935). (Recently it was extended to topological vector spaces (Cauty 2001)). The Tychonoff fixed point theorem states the following. Let $C$ be a nonvoid compact convex subset of a locally convex space and let $f: C \longrightarrow C$ be a continuous map. Then $f$ has a fixed point in $C$. The map $f$ is not assumed to be affine here.

However, for a common fixed point of more than one function, the situation is completely different. Papers (Boyce 1969) and (Huneke 1969) contain independent examples of two commuting maps $f, g$ : $[0,1] \longrightarrow[0,1]$ without a common fixed point. Hence if a common fixed point theorem were to hold, there should be further restrictions on the nature of transformations beyond amenability, and for Day's theorem, this restriction is that the transformations are affine.

The Tychonoff fixed point theorem is being extended in the context of approximate fixed points. For instance, here is one recent elegant result.

Theorem 3.5 (Kalenda 2011). Let $X$ be a Banach space. Then every nonempty, bounded, closed, convex subset $C \subseteq X$ has the weak AFP property with regard to each continuous map $f: C \longrightarrow C$ if and only if $X$ does not contains an isomorphic copy of $\ell^{1}$.

We do not know if a similar program can be pursued for topological groups.

Question 3.6. Does there exist a non-trivial topological group $G$ which has the approximate fixed point property with regard to every continuous action (not necessarily affine) on a bounded, closed convex subset of a locally convex space? of a Banach space?

Question 3.7. The same, for the weak AFP property.

Natural candidates are the extremely amenable groups, see e.g. Pestov (2006). A topological group is extremely amenable if every continuous action of $G$ on a compact space $K$ has a common fixed point. The action does not have to be affine, and $K$ is not supposed to be convex. This is a very strong nonlinear fixed point property.

Some of the most basic examples of extremely amenable Polish groups are:

1. The unitary group $U\left(\ell^{2}\right)$ with the strong operator topology (Gromov and Milman 1983).

2. The group Aut $(\mathbb{Q})$ of order-preserving bijections of the rational numbers with the topology induced from $S_{\infty}$ (Pestov 1998).

3. The group Aut $(X, \mu)$ of measure preserving transformations of a standard Lebesgue measure space equipped with the weak topology. (Giordano and Pestov 2002).

However, at least the group Aut $(\mathbb{Q})$ does not have the AFP property with regard to continuous actions on the Hilbert space. To see this, one can use the same construction as in Proposition 2.8, together with the well-known fact that Aut $(\mathbb{Q})$ contains a closed discrete copy of $\mathbb{F}_{2}$. 


\section{ACKNOWLEDGMENTS}

Cleon S. Barroso is currently as a visiting researcher scholar at the Texas A\&M University. He takes the opportunity to express his gratitude to Prof. Thomas Schlumprecht for his support and friendship. Also, he acknowledges Financial Support form Coordenação de Aperfeiçoamento de Pessoal de Nível Superior (CAPES) by the Science Without Bordes Program, PDE 232883/2014-9. Brice R. Mbombo was supported by Fundação de Amparo à Pesquisa do Estado de São Paulo (FAPESP) postdoctoral grant, processo 12/20084-1. Vladimir G. Pestov is a Special Visiting Researcher of the program Science Without Borders of CAPES, processo 085/2012.

\section{REFERENCES}

AL-GADID Y, MBOMBO B AND PESTOV V. 2011. Sur les espaces test pour la moyennabilité. CR Math Acad Sci Soc R Can 33: $65-77$.

BABENKO I AND BOGATYI SA. 2011. The amenability of the substitution group of formal power series. Izv Math 75: $239-252$. BARROSO CS. 2009. The approximate fixed point property in Hausdorff topological vector spaces and applications. Discrete Cont Dyn Syst 25: 467-479.

BARROSO CS AND LIN PK. 2010. On the weak approximate fixed point property. J Math Anal Appl 365: 171-175.

BARROSO CS, KALENDA OFK AND LIN PK. 2012. On the approximate fixed point property in abstract spaces. Math Z 271: 1271-1285.

BARROSO CS, KALENDA OFK AND REBOUÇAS MP. 2013. Optimal approximate fixed point result in locally convex spaces. J Math Appl 401: 1-8.

BOURBAKI N. 1963. Intégration. Hermann, Paris.

BOYCE WM. 1969. Commuting functions with no common fixed point. Trans Amer Math Soc 137: 77-92.

BROUWER LEJ. 1911. Uber Abbildung von Mannigfaltigkeiten. Math Ann 71: 97-115.

CAUTY R. 2001. Solution du problème de point fixe de Schauder. Fund Math 170: 231-246.

DAY M. 1961. Fixed-point theorems for compact sets. Illinois J Math 5: 585-590.

DE LA HARPE P. 1973. Moyennabilité de quelques groupes topologiques de dimension infinie. CR Acad Sci Paris, Sér A 277: $1037-1040$.

DE LA HARPE P. 1979. Moyennabilité du groupe unitaire et propriété de Schwartz des algèbres de von Neumann. Lecture Notes in Math 725: 220-227.

DOBROWOLSKI T AND MARCISZEWSKI W. 1997. Rays and the fixed point property in noncompact spaces. Tsukuba J Math 21: 97-112.

EDELSTEIN M AND KOK-KEONG T. 1994. Fixed point theorems for affine operators on vector spaces. J Math Anal Appl 181: 181-187.

FLORET K. 1980. Weakly Compact Sets. Lecture Notes in Math. 801, Springer-Verlag, Berlin? Heidelberg ? New York.

GIORDANO T AND PESTOV V. 2002. Some extremely amenable groups. CR Acad Sci Paris Sér I 4: 273-278.

GRIGORCHUK R AND DE LA HARPE P. IN PRESS. Amenability and Ergodic Properties of Topological groups: From Bogolyubov onwards.

GROMOV M AND MILMAN VD. 1983. A topological application of the isoperimetric inequality. Amer J Math 105(4): 843-854. HADŽIĆ O. 1996. Almost fixed point and best approximations theorems in $H$-spaces. Bull Austral Math Soc 53: 447-454.

HAHN F. 1967. A fixed-point theorem. Math Systems Theory 1: 55-57.

HAZEWINKEL M AND VAN DE VEL M. 1978. On almost-fixed-point theory. Canad J Math 30: 673-699.

HUNEKE JP. 1969. On common fixed points of commuting continuous functions on an interval. Trans Amer Math Soc 139: 371-381.

IDZIK A. 1988. Almost fixed point theorems. Proc Amer Math Soc 104: 779-784.

KAKUTANI S. 1938. Fixed-point theorems concerning bicompact convex sets. Proc Imperial Acad Japan 14: 27-31.

KALENDA OFK. 2011. Spaces not containing $\ell^{1}$ have weak approximate fixed point property. J Math Anal Appl 373: $134-137$. KLEE VL. 1955. Some topological properties of convex sets. Trans Amer Math Soc 78: 30-45.

LIN PK AND STERNFELD Y. 1985. Convex sets with the Lipschitz fixed point property are compact. Proc Amer Math Soc 93: 33-39. 
MARKOV A. 1936. Quelques théorèmes sur les ensembles abéliens. Dokl Akad Nauk SSSR (NS:) 10: 311-314.

NOWAK B. 1979. On the Lipschitzian retraction of the unit ball in infinite dimensional Banach spaces onto its boundary. Bull Acad Polon Sci Sr Sci Math 27: 861-864.

PARK S. 1972. Almost fixed points of multimaps having totally bounded ranges. Nonlinear Anal 51: 1-9.

PATERSON AT. 1988. Amenability. University Math. Surveys and Monographs 29, Amer Math Soc, Providence, RI.

PESTOV V. 1998. On free actions, minimal flows, and a problem by Ellis. Trans of the American Mathematical Society 350 : 4149-4165.

PESTOV V. 2006. Dynamics of Infinite-dimensional Groups: The Ramsey-Dvoretzky-Milman phenomenon. University Lecture Series, vol. 40, Amer Math Soc, Providence, RI.

SCARF H. 1967. The approximation of fixed points of a continuous mapping. SIAM J Applied Math 15: 1328-1343.

SCHAUDER J. 1930. Der Fixpunktsatz in Functionalraumen. Studia Math 02: 171-180.

TYCHONOFF A. 1935. Ein Fixpunktsatz. Math Ann 03: 767-776. 